\title{
Early pregnancy maternal cardiovascular profiling in the prediction of hypertensive disease in pregnancy
}

\author{
Jennifer Hogan, Vicky O’Dwyer, Nadine Farah, Mairead M. Kennelly, \\ Michael J. Turner, Bernard Stuart
}

UCD Centre for Human Reproduction, Coombe Women and Infants University Hospital, Dublin, Ireland

Email: jenniferlkhogan@hotmail.com

Received 21 December 2013; revised 15 January 2014; accepted 22 January 2014

Copyright (c) 2014 Jennifer Hogan et al. This is an open access article distributed under the Creative Commons Attribution License, which permits unrestricted use, distribution, and reproduction in any medium, provided the original work is properly cited. In accordance of the Creative Commons Attribution License all Copyrights (C) 2014 are reserved for SCIRP and the owner of the intellectual property Jennifer Hogan et al. All Copyright (C) 2014 are guarded by law and by SCIRP as a guardian.

\section{ABSTRACT}

Prediction of hypertensive disease in pregnancy remains challenging. Risk factor based screening utilizing history, physical characteristics, uterine artery Doppler and serum markers have been used with varying success. As hypertension in pregnancy appears to be associated with changes in the maternal vasculature, we have investigated the utility of maternal arterial elasticity measurement, prior to 20 weeks gestation, in the prediction of hypertensive disease in pregnancy. A HDI/Pulsewave CR2000 Research Cardiovascular Profiling System was used to obtain radial artery pulse pressure waveforms from 623 Caucasian women at the time of their first antenatal visit. Outcome data were available in 610 cases and these comprised the study group. The results showed that women who subsequently developed gestational hypertension $(n=20)$ had higher baseline systolic, diastolic and pulse pressure values (though within the normal range) but similar arterial elasticity to those patients who did not develop hypertension. Women who later developed pre-eclampsia $(n=23)$ had, in addition, reduced large artery elasticity and increased systemic vascular resistance compared with women who did not develop hypertension. The measurement of maternal radial artery elasticity in early pregnancy may provide an additional refinement in screening for hypertensive disease, particularly pre-eclampsia, in pregnancy.

\section{KEYWORDS}

Pre-Eclampsia; Gestational Hypertension; Arterial Wall Elasticity; Pulse Pressure Wave Analysis

\section{INTRODUCTION}

Antenatal care is aimed at the early detection of compli- cations that threaten maternal or fetal well-being in order to monitor these pregnancies more frequently and intervene as necessary [1]. Pre-eclampsia and gestational hypertension are major causes of morbidity and mortality and complicate one in ten of pregnancies, but usually become clinically apparent only in the late second or third trimesters after the disease process is already established [1-3]. This late presentation hinders any opportunities for prevention of pre-eclampsia or gestational hypertension.

If these hypertensive conditions could be predicted earlier, pregnancies at increased risk could be identified and treatment institiuted to reduce the severity of complications [1]. Various strategies such as risk factor based screening, uterine artery Doppler studies and the measurement of serum markers in the first and second trimester, have been evaluated in predicting the risk of preeclampsia, with varying degrees of success $[1,3,4]$.

The etiology of pre-eclampsia remains unknown but it is frequently associated with defective placentation [5]. Abnormal trophoblastic invasion of the maternal spiral arteries is one of the features of pre-eclampsia [4,5]. In normal pregnancy, the spiral arteries change from low capacitance high resistance vessels into high capacitance low resistance vessels through trophoblastic invasion of the muscular wall in the second trimester [6]. In preeclampsia, there is defective trophoblastic invasion and the spiral arteries remain as high resistance vessels [4,5].

As the vessel wall appears to contribute to decreased arterial elasticity in cardiovascular conditions outside of pregnancy, it may also have a key role in vascular disease in pregnancy [7]. The aim of this prospective crosssectional study was to examine the utility of maternal arterial wall elasticity values in early pregnancy (using the diastolic phase of the cardiac cycle) in the prediction of hypertension states in pregnancy. 


\section{SUBJECTS AND METHODS}

White European women were recruited at the time of their first visit to the hospital after sonographic confirmation of an ongoing singleton pregnancy. Parity and age were not considered for the purposes of inclusion in the study. All were recruited at or before seventeen completed weeks of gestation except for one who was recruited before twenty completed weeks of gestation. Women were excluded from this study if they had diabetes or known vascular disease. All women gave written informed consent as approved by the Hospital Ethics Committee and all were given an information leaflet.

After recruitment, all subjects had their cardiovascular system assessed under controlled conditions (semi-recumbent after a period of rest and abstinence from smoking, coffee and talking) using radial artery pulse pressure wave analysis obtained with the HDI/Pulse-wave ${ }^{\mathrm{TM}} \mathrm{CR}$ 2000 Research Cardiovascular Profiling System (HDI Diagnostics, Inc., Eagan, MN, USA). Each pulse pressure wave measurement took approximately ten minutes to complete including the data collection and administrative components. The recording sensor is illustrated in position in Figure 1 while a representative pulse pressure waveform if shown in Figure 2.

Demographic characteristics and pulse pressure wave analysis parameters were entered into a computer database at the time of assessment. Data on pregnancy outcome were obtained from examination of individual patient notes, labour ward records and the hospital's delivery database. Gestation at delivery, mode of delivery and induction of labour were noted.

The development of hypertension in pregnancy for the purpose of the study was determined by reviewing the patient notes and the hospital's delivery database. Preeclampsia was defined as hypertension (systolic blood pressure $\geq 140 \mathrm{mmHg}$ and a diastolic blood pressure $\geq$ $90 \mathrm{mmHg}$ ) after gestational week 20, returning to normal postpartum, with associated proteinuria ( $>300 \mathrm{mg}$ /day of urinary protein). Gestational hypertension was defined as new onset hypertension without proteinuria. Pulse pressure wave analysis parameters were then compared between women with a normal pregnancy and women with hypertensive disorders in pregnancy.

Statistical analysis was performed using SAS Version 9.1 (SAS Institute, Carey, NC 27513-2414, USA). The purpose of the prospective cross-sectional study was to determine if pulse pressure waveform analysis measurements in early pregnancy could predict the subsequent development of hypertensive disease in pregnancy. Demographics and parameters were expressed as mean +/SD and range. A two-sample t-test was used to examine the hypothesis that arterial elasticity in early pregnancy was different in normal pregnancies to pregnancies that were subsequently complicated by hypertension (pre-

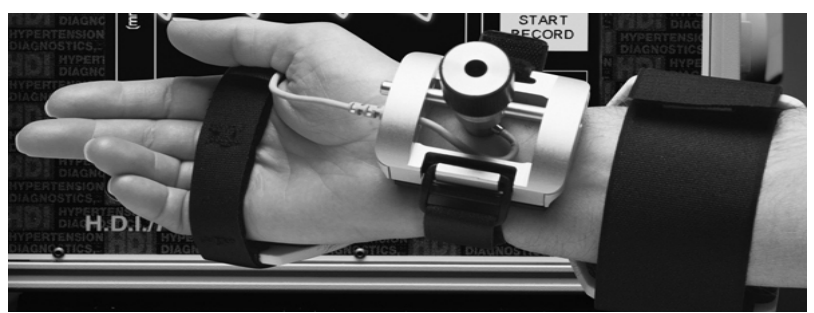

Figure 1. The pulse pressure wave acquisition system.

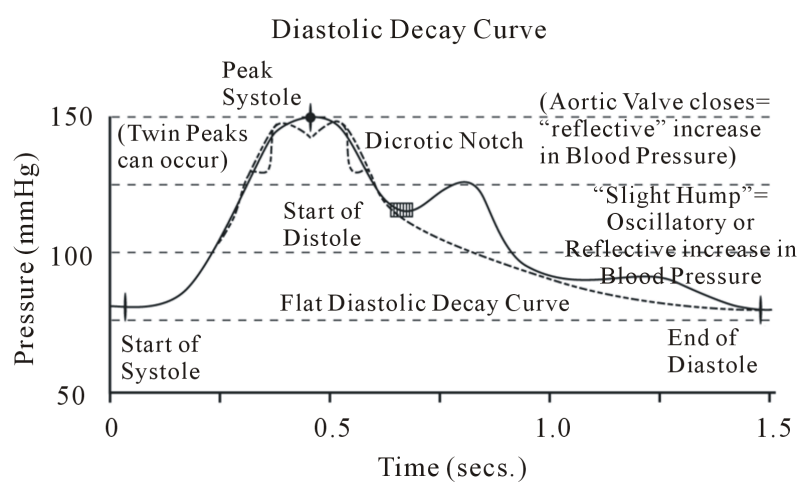

Figure 2. The arterial pulse waveform (Reproduced with permission from HDI Diagnostics, Inc., Eagan, MN, USA).

eclampsia or gestational hypertension). A p value $<0.05$ were considered significant.

Factors such as maternal age, smoking and parity are known to be possible confounders when studying hypertension in pregnancy. Logistic regression was performed to determine the probability of Body Mass Index, maternal age, smoking status or parity having an effect on the pulse pressure-wave measurements.

\section{RESULTS}

Six hundred and twenty three women were recruited. Fifty two percent $(\mathrm{n}=324)$ were primiparous and $48 \%$ (n = 299) were multiparous. Thirteen women miscarried or delivered elsewhere. As outcome data was not available for these cases they were excluded from the analysis resulting in a study cohort of 610 women. Maternal characteristics are presented in Table 1 . There is a wide maternal age range giving representation from all age categories of childbearing age. The BMI range is also wide making the results applicable to a general obstetric population in any large centre.

The baseline cardiovascular parameters are displayed in Table 2. The mean blood pressure measurements as well as the other cardiovascular parameters at this booking visit were within the normal range. This highlights that this population was assumed to be normal from a cardiovascular perspective.

Of the 610 women, 23 (3.8\%) developed pre-eclampsia and 20 (3.3\%) developed gestational hypertension. The results of the pulse pressure wave analysis in these 
Table 1. Characteristics of the study population at the baseline visit $(n=610)$.

\begin{tabular}{ccc}
\hline & Mean $\pm \mathrm{SD}$ & Range \\
\hline Age (years) & $28.2 \pm 5.3$ & $18.0-42.0$ \\
Weight $(\mathrm{kg})$ & $67.5 \pm 13.9$ & $41.0-140.0$ \\
Height $(\mathrm{cm})$ & $164.4 \pm 6.3$ & $140.0-188.0$ \\
Body Mass Index $\left(\mathrm{kg} / \mathrm{m}^{2}\right)$ & $25.0 \pm 4.9$ & $15.5-48.3$ \\
Gestation (weeks) & $12.6 \pm 1.8$ & $6.3-20.0$ \\
Parity & $0.8 \pm 1.0$ & $0-9$ \\
\hline
\end{tabular}

Table 2. Mean baseline cardiovascular parameters of study population $(n=610)$.

\begin{tabular}{ccc}
\hline & Mean \pm SD & Range \\
\hline Systolic blood pressure (mmHg) & $115.0 \pm 9.5$ & $89.0-148.0$ \\
Diastolic blood pressure (mmHg) & $63.4 \pm 7.2$ & $41.0-86.0$ \\
Mean arterial pressure (mmHg) & $81.5 \pm 7.5$ & $57.0-107.0$ \\
Pulse rate (bpm) & $75.5 \pm 9.8$ & $51.0-109.0$ \\
Pulse pressure (mmHg) & $51.5 \pm 6.9$ & $33.0-97.0$ \\
Cardiac output (l/min) & $5.6 \pm 0.6$ & $4.0-8.0$ \\
$\begin{array}{c}\text { Stroke volume (ml/beat) } \\
\text { Small artery elasticity } \\
(\text { ml/mmHg } \times 100)\end{array}$ & $74.7 \pm 11.7$ & $39.0-104.5$ \\
$\begin{array}{c}\text { Large artery elasticity } \\
(\text { ml/mmHg } \times 10)\end{array}$ & $7.7 \pm 2.5$ & $2.3-17.3$ \\
$\begin{array}{c}\text { Systemic vascular resistance } \\
(\text { dyne.sec.cm }\end{array}$ ) & $115.0 \pm 4.1$ & $4.4-34.2$ \\
$\begin{array}{c}\text { Total vascular resistance } \\
(\text { dyne.sec.cm }\end{array}$ & $117.4 \pm 31.8$ & $54.0-312.0$ \\
\hline
\end{tabular}

groups and in the 577 patients (92.9\%) who did not develop hypertension in pregnancy are shown in Table 3.

Some of the cardiovascular measurements (systolic blood pressure, diastolic blood pressure, mean arterial pressure and pulse pressure) are seen to be normal in all groups by standard definitions but the parameters are significantly higher in the groups that developed cardiovascular disease (gestational hypertension and pre-eclampsia) during pregnancy. This highlights that these women have subtle differences in their cardiovascular systems early in pregnancy that may not be apparent with conventional hospital equipment and testing.

Large artery elasticity did not differ between normal women and those women who developed gestational hypertension. Large artery elasticity did however differ between normal women and those women who developed pre-eclampsia $(\mathrm{p}=0.006)$. Small artery elasticity did not differ between the groups. Systemic vascular resistance differed between the women who developed pre-eclampsia and the normal women.
Table 3. Arterial findings in early pregnancy: Normal pregnancy compared to pregnancies later complicated by hypertension ( $\mathrm{p}$ values are in comparison to normal pregnancy values).

\begin{tabular}{|c|c|c|c|}
\hline & $\begin{array}{l}\text { Normal } \\
\text { Pregnancy } \\
(\mathbf{n}=567)\end{array}$ & $\begin{array}{l}\text { Pre-eclampsia } \\
\quad(n=23)\end{array}$ & $\begin{array}{c}\text { Gestational } \\
\text { Hypertension } \\
(\mathbf{n}=\mathbf{2 0})\end{array}$ \\
\hline $\begin{array}{l}\text { Systolic blood } \\
\text { pressure } \\
\text { (mmHg) }\end{array}$ & 114.7 & $\begin{array}{c}124.4 \\
(\mathrm{p}=0.002)\end{array}$ & $\begin{array}{c}125.3 \\
(\mathrm{p}<0.001)\end{array}$ \\
\hline $\begin{array}{l}\text { Diastolic blood } \\
\text { pressure } \\
\text { (mmHg) }\end{array}$ & 63.3 & $\begin{array}{c}69.0 \\
(p=0.006)\end{array}$ & $\begin{array}{c}67.0 \\
(p=0.043)\end{array}$ \\
\hline $\begin{array}{c}\text { Mean arterial } \\
\text { pressure } \\
(\mathrm{mmHg})\end{array}$ & 81.4 & $\begin{array}{c}88.8 \\
(p=0.006)\end{array}$ & $\begin{array}{c}86.2 \\
(p=0.011)\end{array}$ \\
\hline $\begin{array}{l}\text { Pulse pressure } \\
\text { (mmHg) }\end{array}$ & 51.5 & $\begin{array}{c}55.4 \\
(p=0.03)\end{array}$ & $\begin{array}{c}58.4 \\
(p=0.021)\end{array}$ \\
\hline $\begin{array}{l}\text { Large artery } \\
\text { elasticity } \\
(\mathrm{ml} / \mathbf{m m H g} \times 10)\end{array}$ & 15.1 & $\begin{array}{c}11.7 \\
(p=0.006)\end{array}$ & $\begin{array}{c}13.1 \\
(p=N S)\end{array}$ \\
\hline $\begin{array}{c}\text { Small artery } \\
\text { elasticity } \\
(\mathrm{ml} / \mathbf{m m H g} \\
\times 100)\end{array}$ & 7.8 & $6.5(\mathrm{p}=\mathrm{NS})$ & $7.2(p=N S)$ \\
\hline $\begin{array}{c}\text { Systemic } \\
\text { vascular } \\
\text { resistance } \\
\text { (dyne.sec.cm-) }\end{array}$ & 1156.0 & $\begin{array}{c}1341.0 \\
(p=0.032)\end{array}$ & $\begin{array}{l}1188.0 \\
(p=N S)\end{array}$ \\
\hline
\end{tabular}

\section{DISCUSSION}

While gestational hypertension is a relatively benign condition, pre-eclampsia is associated with significant maternal and fetal morbidity up to, and including death [8]. The detection of women at risk for hypertension and pre-eclampsia is one of the main aims of antenatal care though the efficacy of currently available screening programs and treatments is limited [9]. A recent systematic review found that the presence of anticardiolipin antibodies, of bilateral diastolic notches during Doppler ultrasonography of the uterine arteries in mid pregnancy, and urinary kalikrein measurement had moderate predictive accuracy in low risk women but with only a minimal increase in the pre-test probability [10]. These tests were, therefore, found to be of limited clinical use and the review concluded that there was no clinically useful screening test to predict the development of pre-eclampsia [10].

In thisstudy we compared pulse pressure wave analysis in early pregnancy between women with uncomplicated pregnancy and women who later developed hypertensive disorders of pregnancy. We found subtle changes in the cardiovascular system are apparent early in pregnancy (at sixteen weeks or earlier) in women who later develop hypertensive disease. Systolic, diastolic and 
mean arterial pressure were all found to be higher in early pregnancy in those women who went on to have pregnancies complicated by hypertension although the actual values were within the normal range. In addition, large artery elasticity, systemic vascular resistance and total vascular resistance were also found to be different in those pregnancies compared to the normal, with the women who subsequently developed pre-eclampsia having decreased large artery elasticity compared to both the patients who developed gestational hypertension and the normal pregnancy group.

Many of the features of pre-eclampsia (increased capillary permeability, platelet aggregation, increased vascular tone) are also found in association with damage to the vascular endothelium and pre-eclampsia may, therefore, represent an endothelial disorder [11]. It is known that women with pre-existing diseases that are associated with endothelial dysfunction (diabetes, essential hypertension) are at increased risk of developing pre-eclampsia [12]. Other risk factors for cardiovascular disease (e.g. obesity) are also risk factors for hypertension and preeclampsia in pregnancy [12]. This study explored the possibility that these women also showed altered vascular elasticity and resistance in early pregnancy, possibly as a consequence of low-grade endothelial dysfunction. The finding of altered vascular function in early pregnancy in women who subsequently develop pre-eclampsia could also explain the higher incidence of subsequent hypertension in these patients, as they may already have pre-clinical vascular disease exhibited as reduced arterial elasticity and increased peripheral resistance as shown in this study.

Our findings are of interest as they give information about the arterial vessel wall where some of the pathology of pre-eclampsia may originate as opposed to the crude blood pressure measurement or even measurements of blood flow in the vasculature used by other researchers as screening tools for hypertension. These results are consistent with the finding of the higher augmentation index in the pulse pressure wave using systolic phase analysis in pre-eclamptic pregnancies previously reported [13]. With differences in the vasculature being clinically apparentonwith pulse pressure wave analysis in early pregnancy, women at increased risk of developing pre-eclampsia could be selected for more intensive antenatal monitoring or therapy (when developed) aimed at the prevention of the disease by detecting women at risk of pre-eclampsia in the first trimester of pregnancy as opposed to the second trimester when the pathological changes of pre-eclampsia are already established and may be irreversible.

When possible confounding variables such as age, smoking status, parity and BMI were examined (known risk factors for the development of pre-eclampsia), none of these apart from parity were found to have an influence on the subsequent development of pre-eclampsia.

\section{CONCLUSIONS}

To our knowledge, this study is the largest one to date pulse pressure wave analysis in pregnancy using the diastolic portion of the cardiac cycle. Arterial pulse pressure wave analysis is a non-invasive technique that is easy to learn and can be used in the out-patient setting. Use of this technology during routine antenatal care could aid in the identification of women at risk of preeclampsia with associated perinatal and maternal morbidity and mortality.

These results also add to our understanding of preeclampsia at the level of the arterial vessel wall and highlight that the microscopic pathological changes in the vasculature may have implications for both preventative strategies and therapies.

\section{REFERENCES}

[1] Poon, L.C., Kametas, N.A., Maiz, N., Akolekar, R. and Nicolaides, K.H. (2009) First-trimester prediction of hypertensive disorders in pregnancy. Hypertension, 53, 812818.

http://dx.doi.org/10.1161/HYPERTENSIONAHA.108.12 $\underline{7977}$

[2] (2012) Coombe Women and Infant’s University Hospital Annual Clinical Report, 43.

[3] Conde-Agudelo, A. and Belizan, J.M. (2000) Risk factors for pre-eclampsia in a large cohort of Latin American and Caribbean women. BJOG, 107, 75-83. http://dx.doi.org/10.1111/j.1471-0528.2000.tb11582.x

[4] Cudihy, D. and Lee, R.V. (2009) The pathophysiology of pre-eclampsia: Current clinical concepts. Journal of $\mathrm{Ob}$ stetrics \& Gynaecology, 29, 576-582. http://dx.doi.org/10.1080/01443610903061751

[5] Redman, C.W. and Sargent, I.L. (2003) Pre-eclampsia, the placenta and the maternal systemic inflammatory response-A review. Placenta, 24, S21-S27.

[6] Redman, C.W. and Sargent, I.L. (2005) Latest advances in understanding preeclampsia. Science, 308, 1592-1594. http://dx.doi.org/10.1126/science.1111726

[7] Cohn, J.N., Hoke, L., Whitwam, W., Sommers, P.A., Taylor, A.L., Duprez, D., Roessler, R. and Florean, N. (2003) Screening for early detection of cardiovascular disease in asymptomatic individuals. American Heart Journal, 146, 679-685. http://dx.doi.org/10.1016/S0002-8703(03)00499-X

[8] Centre for Maternal and Child Enquiries (CMACE) (2011) Saving mothers' lives: Reviewing maternal deaths to make motherhood safer: 2006-08. The eighth report on confidential enquiries into maternal deaths in the United Kingdom. BJOG, 118, 1-203.

[9] Rooney, C. (1992) Antenatal care and maternal health: How effective is it-A review of the evidence. World 
Health Organization.

[10] Conde-Agudelo, A., Villar, J. and Lindheimer, M. (2004) World Health Organization systematic review of screening tests for preeclampsia. Obstetrics \& Gynecology, 104, $1367-1391$.

http://dx.doi.org/10.1097/01.AOG.0000147599.47713.5d

[11] Roberts, J.M., Taylor, R.N., Musci, T.J., Rodgers, G.M., Hubel, C.A. and Mclaughlin, M.K. (1989) Preeclampsia: An endothelial cell disorder. American Journal of Obstetrics \& Gynecology, 161, 1200-1204.

http://dx.doi.org/10.1016/0002-9378(89)90665-0
[12] Briceno-Perez, C., Briceno-Sanabria, L. and Vigil-De Gracia, P. (2009) Prediction and prevention of preeclampsia. Hypertens Pregnancy, 28, 138-155. http://dx.doi.org/10.1080/10641950802022384

[13] Khalil, A.A., Cooper, D.J. and Harrington, K.F. (2009) Pulse wave analysis: A preliminary study of a novel technique for the prediction of pre-eclampsia. BJOG, 116, 268-276.

http://dx.doi.org/10.1111/j.1471-0528.2008.01906.x 